\title{
Programming Matter through Strain
}

\author{
Michael J. Motala ${ }^{\mathrm{a}, \mathrm{b}, \S}$, Daniel Perlitz ${ }^{\mathrm{c},}$, Christopher M. Daly ${ }^{\mathrm{a}, \mathrm{b}}$, Peixi Yuan ${ }^{\mathrm{a}, \mathrm{b}}$, Ralph G. Nuzzo ${ }^{\mathrm{a}, \mathrm{b}^{*}}$, \\ and K. Jimmy Hsia ${ }^{\text {b,c,d,e* }}$ \\ ${ }^{a}$ Department of Chemistry \\ University of Illinois at Urbana-Champaign, Urbana, IL 61801, USA \\ ${ }^{b}$ Frederick Seitz Materials Research Laboratory \\ University of Illinois at Urbana-Champaign, Urbana, IL 61801, USA \\ ${ }^{c}$ Department of Mechanical Science and Engineering \\ University of Illinois at Urbana-Champaign, Urbana, IL 61801, USA \\ ${ }^{d}$ Micro and Nanotechnology Laboratory \\ University of Illinois at Urbana-Champaign, Urbana, IL 61801, USA \\ ${ }^{e}$ Current address: Departments of Mechanical Engineering and Biomedical Engineering \\ Carnegie Mellon University, Pittsburgh, PA 15213, USA

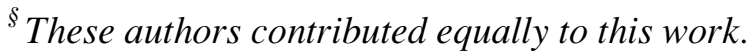

\begin{abstract}
We describe the use of light in a lithographic form of grayscale patterning as a means to program the properties and folding mechanics of flat, thin-film-polymeric materials. In this process, a finely dispersed (phase-separated) mixture of photoresist (SU-8 50) in polydimethylsiloxane (PDMS) is irradiated with ultraviolet light through a photomask. The subsequent photoresist cross-linking in the exposed regions causes changes in the material's chemo-mechanical properties (notably making it stiffer and

*Corresponding authors,E-mail: r-nuzzo@illinois.edu,kjhsia@cmu.edu
\end{abstract}


more resistant to solvent-induced swelling in the area of exposure). Light scattering due to the dispersed, non-index-matched photoresist domains leads to an intrinsic grayscale profile of the pattern width through the depth of the exposure field, a feature bringing significant and previously unexplored consequences for strain-induced folding dynamics. Solvent induced swelling, where PDMS absorbs a nonpolar solvent, is used to actuate folding mechanics with complex temporal and spatial profiles that explicitly follow the design rules established by the gradient cross-link density, features that elicit a programmable swelling (and therefore, folding) of the two-dimensional sheets. During investigation and optimization of the system, we observed an interesting temporal and biomimetic folding phenomenon that distinguishes the current results from other forms of strain induced folding reported in the literature. Under specific fabrication parameters, an evolution through an intermediate metastable state is observed, one in which the material will fold in one direction, then flatten and fold in the opposite direction. Mechanics modeling and finite element simulations have led to a detailed understanding of the system and the dynamics that allow a temporal evolution of 3-D structure through a double mode of folding. Insights into these mechanisms provide an advanced understanding of strain-induced folding in the field of soft materials.

Keywords: mesoscale self-assembly; strain gradients; programmable matter; PDMS; SU8

\section{Introduction}


Mesoscale self-assembly (MESA) involves the extension of molecular selfassembly concepts to the micrometer to millimeter range [1-6]. In MESA, individual components are typically easy to fabricate and offer a broad range of selection for the building process, and the larger size domain allows MESA to be less defect-sensitive than in molecular self-assembly. Currently in the MESA design space, there is interest in the biomimetic formation of 3-D objects self-assembled via folding of a 2-D precursor [712]. These folding materials typically rely on well-developed planar micro fabrication techniques that are most commonly used to incorporate electronic forms of functionality into a material. These capabilities provide a powerful approach to patterning materials with attributes exploitable within MESA.

Many driving forces have been used to manipulate the 3-D folding assembly process, including magnetic force [8], capillary interaction [12], surface tension [7, 1314], epitaxial lattice mismatch [15-17], and swelling strain [11, 18]. These systems generally have one possible folded configuration, and variation of the design to induce multiple folding configurations is often difficult.

In this work, we use light to "program" the folding properties of a flat twodimensional composite material using photolithography. The composite material is composed of PDMS a flexible silicone elastomer that swells in nonpolar solvents (i.e. toluene, hydrocarbons, chlorinated solvents) $[19,20]$, and $\mathrm{SU}-8$, a photoresist that is insoluble in nonpolar solvents and forms a hard, mechanically robust material upon ultraviolet cross-linking. In the current work, mixtures of PDMS and the light sensitive agent, SU-8, are spin coated onto a polymer support and heated to cure the PDMS component, creating the biphasic material precursor that can be differentially treated to 
vary the folding configuration. Exposure of the composite film to UV-light is the critical "programming" step that allows for facile access to different folding structures. Under UV irradiation, the SU-8 component on the surface of the exposed portions of the material is selectively cross-linked. As a result of stochastic light scattering and absorption of UV light in the material, a gradient of cross-linked SU-8 concentration is formed starting at the exposed surface and dissipating outward and downward into the bottom and unexposed portions of the material.

After curing the SU-8 programming agent, the composite material is immersed in nonpolar solvent, causing the material to differentially swell. The non-exposed regions swell more as the PDMS component absorbs the nonpolar solvent. The light-exposed regions inhibit swelling due to the high concentration of cross-linked SU-8 component. This disparity in swelling behaviors induces differential strain in the system, which results in deformation of a two-dimensional sheet and spontaneous curvilinear folding in a specific and, as we show, predictable fashion. One of the distinct and exciting folding phenomena observed in this investigation is that of double folding, where, for a specific set of design rules, the PDMS-SU-8 sample folds in one direction initially, unfolds and then refolds in a different direction. The latter dynamics demonstrate an interesting biomimetic phenomenon that has not been broadly studied in soft-material mechanics to date.

In this work, we report mechanistic modeling and finite element simulation efforts that fully simulate the experimentally observed folding mechanics of this material. Such mechanics modeling tools can be used to design simple substrates that fold into complex, non-planar, geometries. The deformation of thin sheets due to gradients in strain has been 
studied with a variety of analytical and computational models. Past work in the field has focused on using energetic modeling [21-26] and computational studies [15, 23-24, 27 28 ] to analyze folding modes of bilayer sheets with varying levels of normal strain mismatch. Recently, Stoychev et al. [24] modeled the progression of swelling ratio through the volume of polymer sheets with a heat transfer model. Because heat transfer is governed by similar differential equations, they could use this model to understand how these sheets deform. Although past work has been done to study deformation modes in thin polymer systems, further work is necessary to understand how complex gradients in swelling ratios throughout a sheet's volume affect overall deformation modes. These features are addressed quantitatively in the current work.

\section{Experimental Method}

\subsection{Materials Used}

Polydimethylsiloxane (PDMS, Dow Corning, Sylgard 184), SU-8 50 photoresist (Micro Chem, 25-50 wt \%), glass slide (Fisher), and Kapton tape (CS Hyde).

Nonpolar Solvents: Pentane, Toluene, 2-Butanone, Ethyl acetate, and Dioxane (SigmaAldrich). For photoexposure and mask alignment, a Karl Suss MJB3 Contact Mask Aligner was used.

\subsection{Fabrication of Patterned Sample}

Pre-gel monomer and initiator of PDMS were mixed (10:1 monomer:initiator molar ratio). SU-8 photoresist was added to the PDMS pre-gel mixture (Between 25\% and $50 \%$ SU-8 by wt), resulting in an opaque white mixture (higher mass ratios of SU-8 do not spin coat well). The mixture is allowed to degas for 30 min under vacuum, followed by spin-coating (between 500-3500 rpm range, 30 seconds, onto a glass slide 
coated with Kapton tape. The material was then heated $\left(70{ }^{\circ} \mathrm{C}, 2 \mathrm{hr}\right)$ to allow the PDMS to cross-link and to remove the solvent from the SU-8 component of the mixture. Following photoexposure (Karl Suss mask aligner, $45 \mathrm{sec}$ exposure) with the desired photomask, the material was again heated (typically $150{ }^{\circ} \mathrm{C}, 10 \mathrm{~min}$ ) to cure the photoactivated SU-8 portions.

\section{Results and Discussion of Analyses of Mechanics}

\subsection{Experimental Observations}

In the present work, we use relatively simple masks (e.g. parallel lines of different widths and spacing), although more complex patterns can be easily achieved. After the post-bake curing of the photoactivated SU-8 portions, the material is removed from the Kapton tape-coated glass slide and then cut into the desired size/shape. Fig. 1 shows exemplary cylindrical shapes fabricated in this way along with a schematical depiction of the experimental setup (Fig. 1a) used to fabricate the structures shown.

(a)
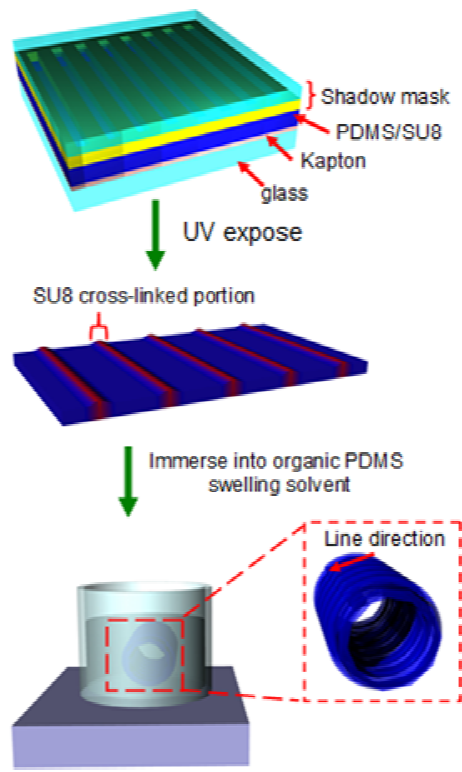

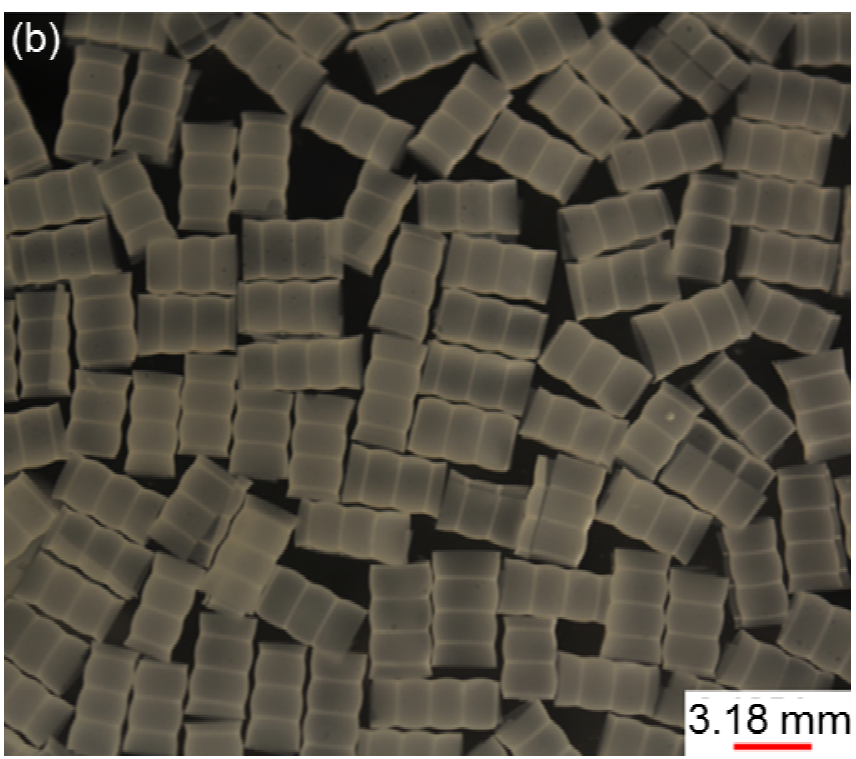

Figure 1. (a) Schematic depiction of the fabrication set-up, light programming step, and then folding process. (b) Image of multiple samples that were programmed to fold into a cylinder. 
In this exemplary demonstration, a photomask design of straight, parallel lines with a width of $10 \mu \mathrm{m}$ and spacing of $200 \mu \mathrm{m}$ leads to monotonic folding of the $\sim 120 \mu \mathrm{m}$ thick sheet in only one limiting/specific direction. The folding in this case results in a rolledup, cylindrical, shape of the sheet (Fig. 1b) in which the folding axis is perpendicular to the cross-linked lines, which face inwards. The specifics of this folding mode are discussed in greater detail below.

3.2 Property and Spatial Characterization of Photoexposed PDMS-SU-8

To examine the cross-linking profile, one that varies through the thickness of the specimens, a PDMS sample with large line spacing was exposed to a dilute solution of Rhodamine 6G. The fluorescent dye absorbs preferentially in the lower cross-linkdensity regions of the PDMS-SU-8 film. As a result, the highly cross-linked regions are much darker when viewed by fluorescence microscopy than the unexposed, less crosslinked regions. Three useful material region labels--heavily cross-linked (XL) section, non-cross-linked (NXL) section, and partially cross-linked (PXL) section-were developed from the exemplary micrograph (Fig. 2a) of the cross-section of the dyed film along with an analysis of the fluorescent cross-sectional image made using MATLAB 
(Fig. 2b, as described in detail in Supplemental Materials Section S1).
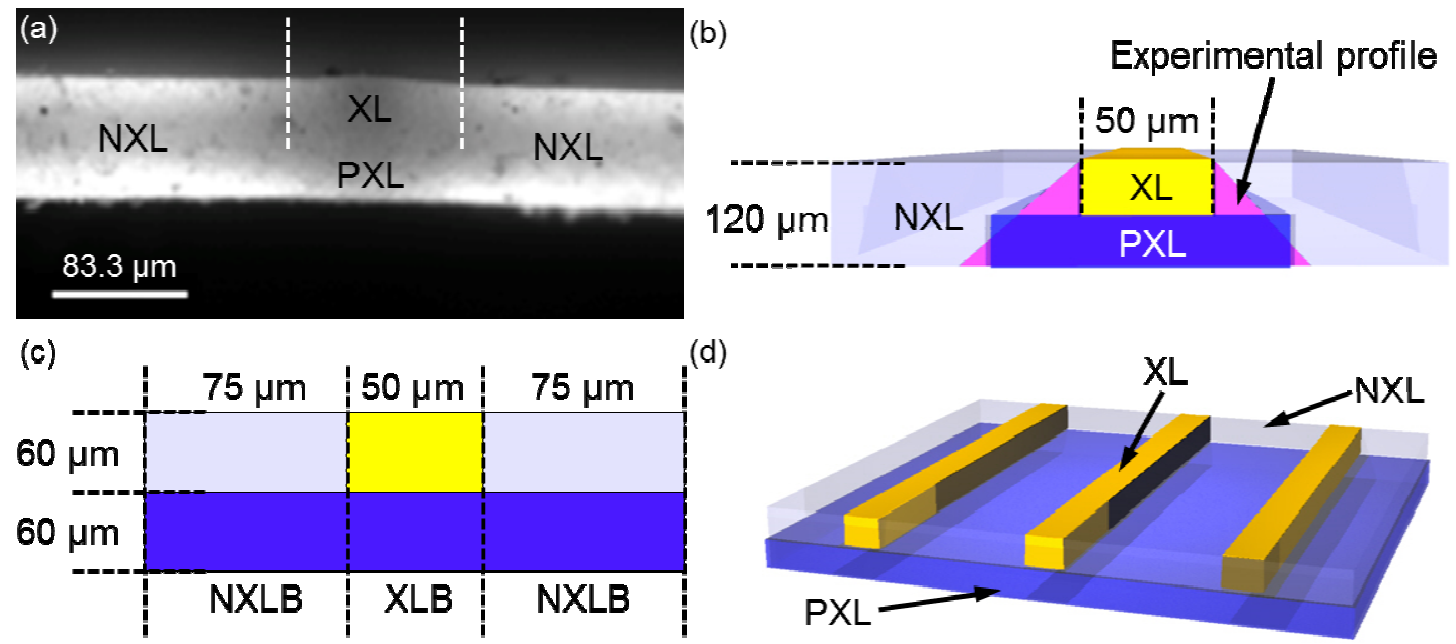

Figure 2. (a) Image of a cross-section of the PDMS-SU8 sample dyed with Rhodamine 6G. The white dashed lines indicate the region of photoexposure through the photomask. (b) Schematic depiction of the XL, PXL, and NXL regions of the gel overlaying the approximate experimental profile (pink) of the SU-8 cross-linking. (c) Final sheet cross-section with regions and XLB, NXLB bilayers labeled. (d) Schematic depiction of a portion of the idealized full sheet system.

The placement of the XL, NXL, and PXL regions shown in Fig. $2 b$ is an idealized depiction for modelling purposes of the more complicated cross-link density distributions through the thin sheet thickness. At low line spacing, it can be approximated, again for modelling purposes, that the PXL sections on the bottom layer overlap, forming a uniform PXL layer across the entire bottom layer. This simplifies the idealized model in our analysis, allowing the sheet to be "divided" into two repeating sections: cross-linked bilayers (XLB) and non-cross-linked bilayers (NXLB), as depicted in Fig. 2c. Due to scattering of the irradiating light, the width of the XL section is notably greater than that of the width of the parallel lines of the photomask.

The materials of the XL, NXL, and PXL sections from Figs. 2b-c can be approximated as linear elastic, isotropic. The PDMS literature value of 0.499 [29] was used for the Poisson's ratio of each section. The swelling ratio, $\lambda$, can be estimated by 
measuring the amount of swelling of a sheet fully exposed to light (XL) and that unexposed to light (NXL). The swelling ratio is defined as

$$
\lambda=l_{f} / l_{i}
$$

where $l_{f}$ is the final length of one side of a swelled sample and $l_{i}$ is the initial length of that side. It is known that highly cross-linked materials have a higher elastic modulus, lower swelling ratio [30], and absorb solvent more slowly [31] than that of the partially cross-linked or non-cross-linked region. The elastic modulus of the PXL and XL sections were measured with atomic force microscopy and the NXL material was assumed to have the literature elasticity value of PDMS [32]. The values of Young's moduli, Poisson's ratios, and swelling ratios used in the analysis are given in Table 1.

Table 1. Materials characteristics of the three separate regions of the of the PDMS-SU-8 material.

\begin{tabular}{c|ccc}
\hline Sheet Section & Elasticity $(\mathrm{MPa})$ & Poisson's Ratio & Swelling Ratio \\
\hline $\mathrm{XL}$ & 15.44 (High) & 0.499 & 1.171 (Low) \\
NXL & 0.75 (Low) & 0.499 & 1.53 (High) \\
PXL & 11.63 (Medium) & 0.499 & {$[-]$ (Medium) } \\
\hline
\end{tabular}

\subsection{Mechanistic Model}

A mechanistic model was developed to study the folding phenomenon in the $2 \mathrm{D}$ thin sheet upon differential swelling. Depending on the analysis, the material properties of each section were varied with the measured properties described in Table 1 serving as a baseline. In all simulations, the qualitative relationships of material properties between the three sections were upheld. Supplemental Materials Section S2 describes the material properties used in each simulation.

\subsection{Finite Element Analysis}


When a patterned 2D sheet of the composite is submerged in a solvent, absorption does not occur uniformly throughout the sample, but exhibits a transient stage with swelling occurring initially from the surfaces and gradually propagating towards the sheet interior. Eventually the swelling is saturated in the material with each section reach its maximum strain uniformly. ABAQUS/Standard finite element analyses were performed to analyze uniform swelling and transient stages. In the uniform swelling analysis, a model of uniform swelling in each section was used to determine the preferred folding mode given a set of elasticity and swelling strain. In the transient analysis the diffusion of solvent as well as material swelling was modeled. For all analyses, the swelling strain was modeled as linear function of solvent concentration. This assumption gives

$$
\varepsilon_{0}=\frac{C}{C_{s}} \varepsilon_{0 s}
$$

where $\varepsilon_{0}$ is the swelling strain, $C$ is the solvent concentration, $C_{S}$ is the concentration of solvent at saturation, and $\varepsilon_{0 s}$ is the swelling strain when the sheet is fully saturated with solvent ( $\varepsilon_{0 s}$ is henceforth known as saturated swelling strain). Solvent diffusion was modeled with Ficks law with a constant diffusion coefficient. Based on these assumptions, we developed an equivalent heat transfer and thermal expansion model in ABAQUS/Standard using the shell element. The model is a common first order approximation for similar systems $[24,28]$. This model is described in detail in Supplemental Materials Section S3.

The exemplary data shown in Fig. 3 illustrate that for a patterning design rule that yields a non-overlapping array of cross-linked lines, a preferred folding mode of two high symmetry possibilities (Figs. 3a- b) is exclusively adopted. 


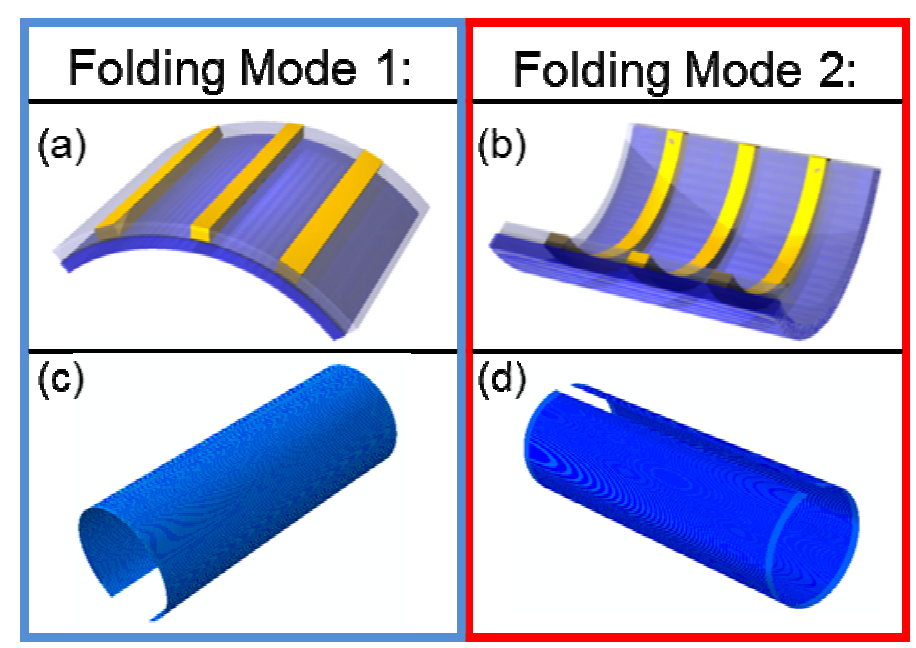

Figure 3. Depictions of the saturated swelling model showing schematics of (a) FM1 (blue outline), (b) FM2 (red outline), and (c, d) the corresponding simulation results.

While folding mode 2 (FM2) is the only experimentally observed saturated monotonic folding mode, our modelling (Figs. 3c-d) suggests that certain photomask designs can give rise to rolled-up cylinders of either folding mode 1 (FM1) (Fig. 3a) or FM2 (Fig. 3b) type, depending on the geometry of the cross-linked line width and spacing as well as swelling ratios realized in different sections. FM1, in contrast to that of FM2, describes a folding mode where the folding axis is parallel to the cross-linked lines, which face outwards. Detailed conditions which favor each of these different monotonic folding modes will be presented in the numerical analysis below with a uniform swelling model.

When the spacing between the cross-linked lines is 50-200 $\mu \mathrm{m}$ (exposed using a $50 \mu \mathrm{m}$ feature width mask), however, instead of monotonic folding, we observe an interesting phenomenon of double folding. The sheets initially fold as described by FM1, but immediately reverse and begin to unroll, and then fold abruptly into the symmetry described in FM2. This form of double folding is a robust process, going from FM1 to FM2 in under a minute. A time sequence of the experimental observation of double folding is shown in Fig. 4a, and a video of the double folding experiment is provided in 
the Supplemental Materials (Video 1). The model simulations corresponding to these experimental observations, depicted in Fig. 4b, are discussed in detail in sections 3.5-6. Videos showing simulation of double folding can be found in the Supplemental Materials (Videos 2 and 3).

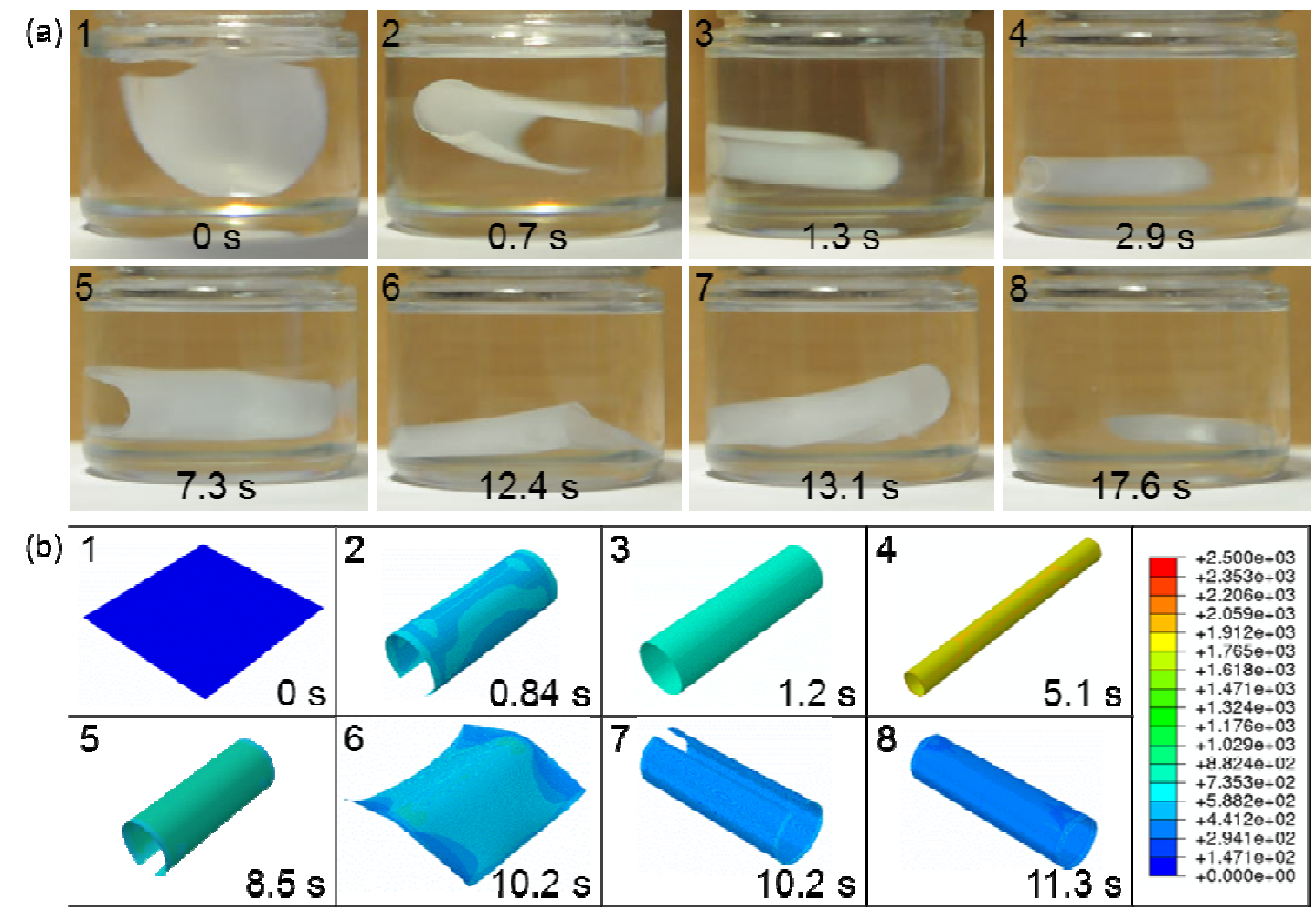

Figure 4. (a) Series of images (a-1 to a-8) showing experimental double folding. (b) A series of schematic representations (b-1 to b-8) of the computational simulation of double folding. The colors of these simulation images give a contour map indicating relative von Mises stress values $(\mathrm{kPa})$, as described by the color legend to the right (see section 3.6 for more discussion of the von Mises stress).

\subsection{FM1 and FM2 Folding in the Saturated Swelling Model}

To understand the mechanisms and identify the conditions of different folding modes driven by differential swelling, we conducted numerical analyses of the mechanical deformation of sheets consisting of patterned cross-linked regions. The idealized model is the periodic structure shown in Fig. 2c above, where the geometric 
parameters are chosen based on the experimental set up. Parametric studies are carried out by systematically changing the swelling ratios and elastic properties of the XL, NXL, and PXL regions. For the purpose of identifying the conditions for different folding modes, uniform swelling within each section is considered.

FM1 and FM2 are both observed outputs for the saturated swelling model depending on the mechanical and swelling properties of the XL, PXL and NXL sections. Fig. 3 depicts the two computationally observed folding directions and corresponding simulation results. This analysis was used to obtain "phase diagrams" of preferred folding modes as functions of different material properties of each section. Fig. 5 shows the map of folding modes as a function of the normalized swelling strain mismatch for the XL, PXL, and NXL sections. Because the swelling of the XL section is always less than that of the PXL section, only the cases above the 45 degree dashed line were considered.

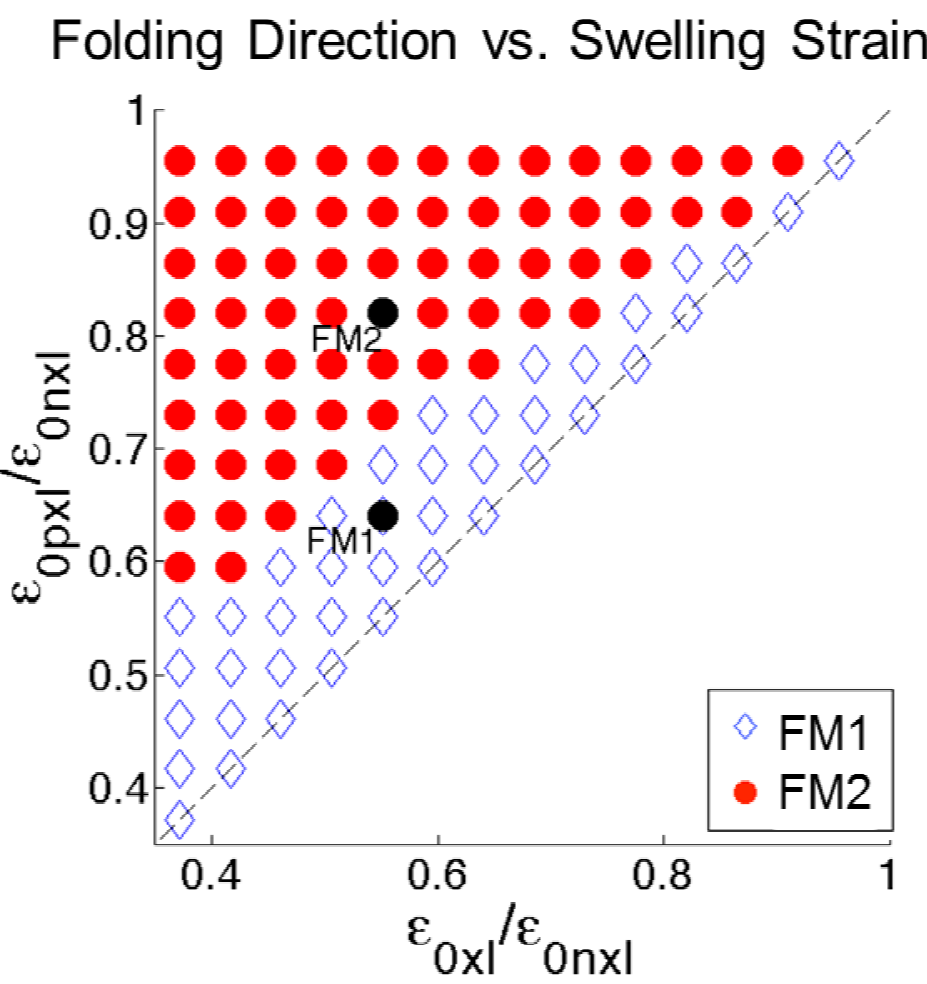

Figure 5. Scatter plot relating folding direction to predefined swelling ratio. 
It is noted that the elastic properties of each material section were held constant throughout these simulations. It was found that the preferred folding modes are very sensitive to the swelling ratio, and less sensitive to the elasticity of different sections (see Supplemental Materials Section S4).

It is known from the classic work by Timoshenko [33] that the bending moment produced by differential swelling in a bilayer is directly proportional to the magnitude of the swelling strain difference between the two layers.

Fig. 5 shows that if the swelling ratios of the XL and PXL materials are relatively close, the sheet exhibits FM1 because the swelling ratio difference in the XLB is not significant enough to drive the folding of the sheet. Instead, the sheet is driven by the swelling ratio difference between the materials in the NXLB, which drives folding away from the exposed side. The sheet folds around the axis parallel to the cross-linked lines because the moment created by the strain difference in the NXLB is not large enough to bend the stiff, highly elastic, XL lines. The sheet exhibits FM2 when the PXL section has a swelling ratio closer to that of the NXL section, leading to a bending moment decrease in the NXLB and increase in the XLB.

\subsection{Transient Analysis of Double Folding}

A transient analysis was carried out to study the double folding phenomenon and identify a likely mechanism for the change in folding direction from FM1 to FM2. In the finite element analysis, three parameters--the solvent diffusion rate, the maximum swelling ratio, and elasticity of each section--were chosen such that double folding occurs in our numerical simulations. In this analysis, elasticity was held constant throughout the simulation. In reality, the elasticity of each material does change temporally throughout 
the folding. Our simulations, however, showed that changing elasticity with the amount of swelling would not have a significant effect on the deformation mode, as is discussed in Supplemental Materials Section S5. Fig. 4b shows the time-lapse simulation results corresponding to the experimental images of the double folding phenomena (Fig. 4a). A movie of the simulation is provided in the Supplemental Materials. The color contours shown in these simulation images correspond to calculated von Mises stress values (color legend, Fig. 4b). Despite the assumptions used in the material property determination for this simulation, these calculated values show a relative stress increase of an order of magnitude as the thin film folds into FM1 (Fig. 4b, 1-4). This substantial von Mises stress is shown to be largely released as the thin film unfolds from FM1 and folds into FM2 (Fig. 4b, 5-8).

In order to more clearly understand the causes of the double folding phenomena, the swelling strain half way through the thickness of each material in the mid-section of the sheet is plotted against simulation time in Fig. 6.

\section{Swelling Strain vs. Simulation Time}

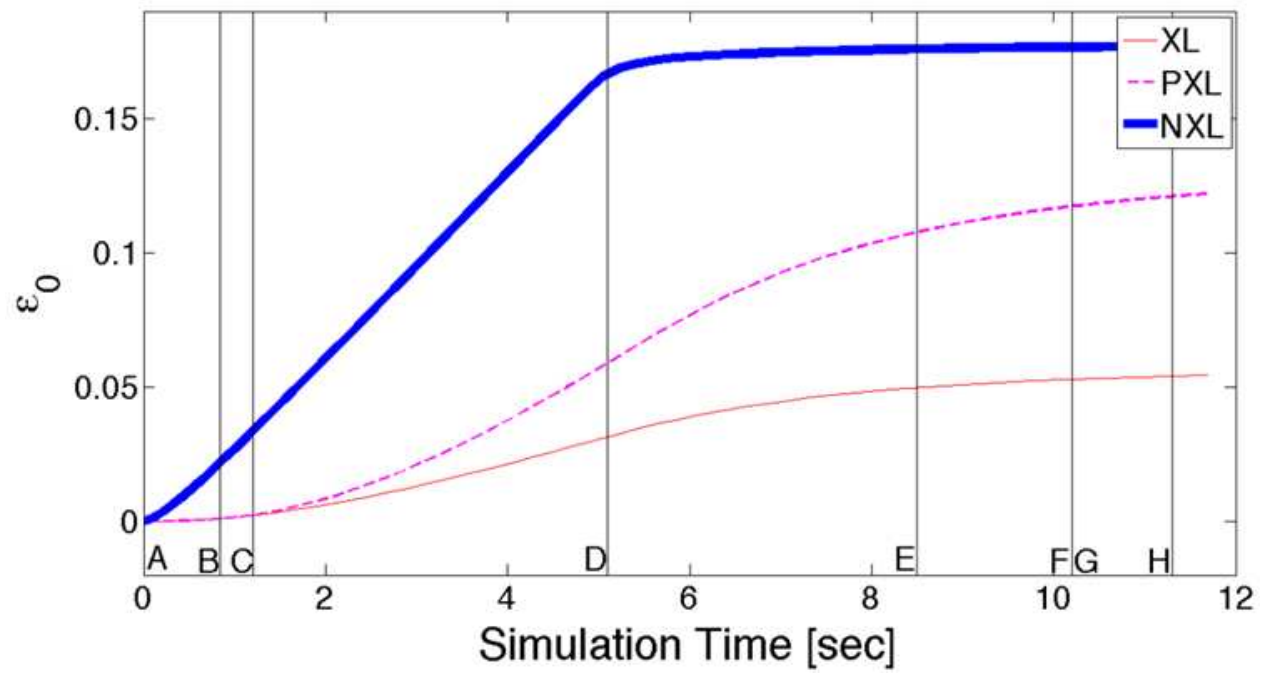

Figure 6. Swelling strain $\left(\varepsilon_{0}\right)$ of each region of the material throughout simulation of the double folding phenomenon. 
The lines labelled A-H in Fig. 6 correspond to the computational times shown in Fig. 4b, 1-8. Fig. 6 shows that, at the beginning of the simulation, the PXL and XL materials have almost the same swelling strain and the swelling strain of the NXL material is much higher. This is because the NXL regions absorb solvent significantly faster than the XL and PXL regions. The large swelling strain difference between materials of the NXLB and the high stiffness of the XL material leads to dynamics adhering to the FM1 mode of action. As the simulation begins, the NXL reaches saturated swelling strain early as the swelling in the PXL and XL materials continues to increase. The increase in PXL swelling strain widens the swelling strain difference between materials of the XLB and decreases the swelling strain difference between materials of the NXLB, causing the sheet to unroll and quickly pass into the FM2 mode of deformation.

\section{7 "Snap" during double folding}

Another important feature of the double folding is the quick snap from the FM1 to FM2 modes of deformation. This action is seen both experimentally and in our numerical simulations (from the time steps), as shown between Fig. 4a-5,6 experimentally and Fig. $4 \mathrm{~b}-6,7$ in the model. The snap phenomenon is also evident in the movies of experimental study and numerical simulations in Supplemental Materials. The snap occurs faster in the computational results, likely because the computational results do not account for softening of each material during folding. Our numerical results indicate that, as the sheet unrolls, the system builds a high level of residual strain energy, which is released when the plate snaps into the new folding mode. This behavior is similar to the snapping of bi-stable domes studied by Madhukar et al [34] or spiral structured interconnects studied by Lv et al [35]. 


\subsection{Complex Programmable Folding Designs}

To demonstrate the versatility of the programmability of this method, more complex folding patterns were explored through different photomask patterns and material shapes. Figs. 7a-c demonstrate three exemplary mask patterns that were tested. Each design shows the photomask (inset) and a corresponding successful computational simulation.

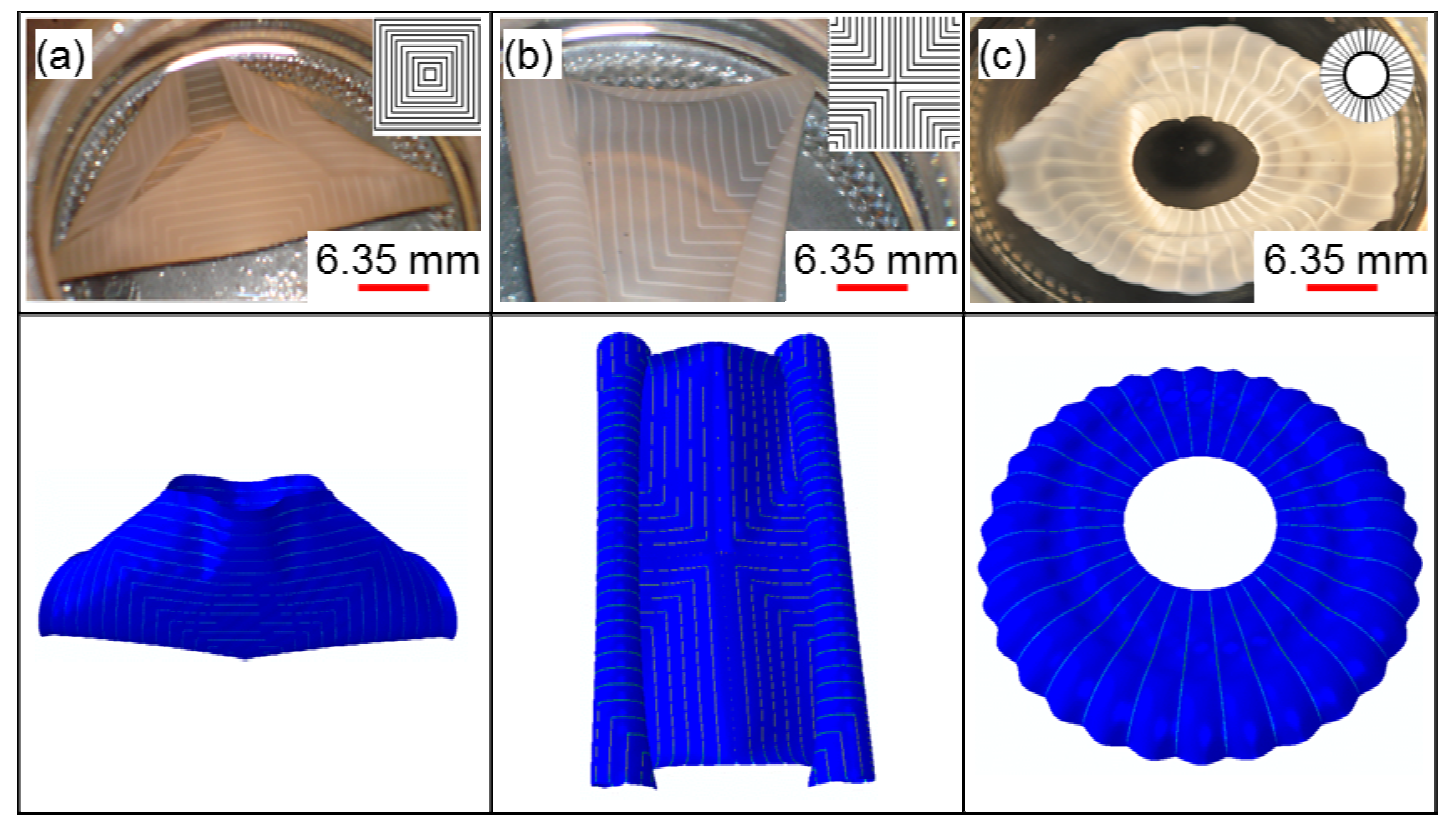

Figure 7. (a-c) Images of three folded samples each programmed with a different photomask design (shown by the inset images) and the computational representations (schematic depiction shown below each image) of each different design.

To successfully simulate such complex deformation, the materials properties of different regions need to be adjusted (Supplemental Materials S6). Better materials property data would allow the computational model to be more quantitatively predictive.

The sample in Fig. 7a uses a photomask with several concentric squares separated by $1000 \mu \mathrm{m}$ spacing. As seen in the image, the corners of this patterned film roll towards the center because the net force at the patterned square directs the folding $45^{\circ}$ to the patterned lines. This sample also folds in half preventing further inward folding of the 
corners. If the patterned lines are directed outward perpendicular to the edges (Fig. 7b), the edges of the film, rather than the corners, are directed to fold inward. The sample folds along two opposite edges and each edge folds into a cylinder. Fig. 7c shows a pinwheel with the patterned lines radiating from the cut out center on a circular film. As in regular folding, each edge folds inwards towards the exposed face, and the rippling occurs to conserve the different circumferences of the two edges.

\section{Conclusion}

We have demonstrated that use of photo-patterning and other simple fabrication tools on composite films of SU-8 and PDMS can control the folding of the patterned thin sheet into 3D curvilinear shapes. The UV-exposed areas resist the swelling when the composite is placed in nonpolar solvent and the differential swelling strains in regions with different UV exposure give rise to different folding behavior. The folding can be controlled through various experimental parameters including, but not limited to, sample composition, sample thickness, mask design, post-bake curing temperature, and organic solvent selection. Furthermore, we have demonstrated that different folding behavior can be predicted through FEM analysis of an idealized model. The parameter space ("phase diagram") that leads to the two dominant folding modes, FM1 and FM2, is mapped out, and the mechanism of the interesting double folding phenomenon is identified. Ultimately, we envision the possibility of programming additional levels of control into the system. This could be achievable through the use of a secondary programming tool after the folding step.

\section{Acknowledgement:}


The authors gratefully acknowledge financial support for the materials development, characterization, and fabrication efforts provided by the U.S. Department of Energy, Office of Basic Energy Sciences, Division of Materials Sciences and Engineering under Award \# DE-FG02-07ER46471, and partial support of the modelling efforts (DP) provided by the National Science Foundation IGERT Grant \# DGE 0965918. 


\section{References:}

1. Boncheva, M.; Whitesides, G. M.. Making things by self-assembly. MRS Bull. 2005, 30(10), 736-742.

2. Boncheva, M.; Bruzewicz, D. A.; Whitesides, G. M. Millimeter-scale selfassembly and its applications. Pure Appl. Chem. 2003, 75(5), 621-630.

3. Cölfen, H.; Mann, S. Higher-order organization by mesoscale self-assembly and transformation of hybrid nanostructures. Angew. Chem. Int. Ed. 2003, 42(21), 2350-2365.

4. Whitesides, G. M.; and Boncheva, M. Beyond molecules: Self-assembly of mesoscopic and macroscopic components. Proc. Natl. Acad. Sci. USA. 2002, 99(8), 4769-4774.

5. Bowden, N. B.; Weck, M.; Choi, I. S.; Whitesides, G. M. Molecule-mimetic chemistry and mesoscale self-assembly. Acc. Chem. Res. 2001, 34(3), 231-238.

6. Xu, R.; Jang, K.-I.; Ma, Y.; Jung, H. N.; Yang, Y.; Cho, M.; Zhang, Y.; Huang, Y.; Rogers, J. A. Fabric-based stretchable electronics with mechanically optimized designs and prestrained composite substrates. Extreme Mech. Lett. 2014, 1, 120-126.

7. Leong, T. G.; Lester, P. A.; Koh, T. L.; Call, E. K.; Gracias, D. H. Surface tension-driven self-folding polyhedra. Langmuir. 2007, 23(17), 8747-8751.

8. Boncheva, M.; Andreev, S. A.; Mahadevan, L.; Winkleman, A.; Reichman, D. R.; Prentiss, M. G.; Whitesides, S.; Whitesides, G. M. Magnetic self-assembly of three-dimensional surfaces from planar sheets. Proc. Natl. Acad. Sci. 2005, 102(11), 3924-3929.

9. Alben, S.; Brenner M. P. Self-assembly of flat sheets into closed surfaces. Phys. Rev. E. 2007, 75(5), 056113-1/8.

10. Brittain, S. T.; Schueller, O. J. A.; Wu, H.; Whitesides, S.; Whitesides, G. M. Microorigami: Fabrication of small, three-dimensional, metallic structures. $J$. Phys. Chem. B. 2001, 105(2), 347-350.

11. Guan, J.; He, H.; Hansford, D. J.; Lee, L. J. Self-folding of three-dimensional hydrogel microstructures. J. Phys. Chem. B. 2005, 109(49), 23134-23137. 
12. Py, C.; Reverdy, P.; Doppler, L.; Bico, J.; Roman, B.; Baroud, C. N. Capillary origami: Spontaneous wrapping of a droplet with an elastic sheet. Phys. Rev. Lett. 2007, $98,156103-1 / 4$.

13. Randall, C. L.; Leong, T. G.; Bassik, N.; Gracias, D. H. 3D lithographically fabricated nanoliter containers for drug delivery. Adv. Drug Deliv. Rev. 2007, 59(15), 1547-1561.

14. Leong, T. G.; Benson, B. R.; Call, E. K., Gracias, D. H. Thin film stress driven self-folding of microstructured containers. Small. 2008, 4(10), 1605-1609.

15. Chun, I. S.; Challa, A.; Derickson, B.; Hsia, K. J.; Li, X. Geometry Effect on the Strain-Induced Self-Rolling of Semiconductor Membranes. Nano Lett. 2010, 10(10), 3927-3932.

16. Froeter, P.; Yu, X.; Huang, W.; Du, F.; Li, M.; Chun, I.; Kim, S. H.; Hsia, J. K.; Rogers, J. A.; Li, X. 3D hierarchical architectures based on self-rolled-up silicon nitride membranes, Nanotechnology. 2013, 24(47), 475301.

17. Clause, A. M.; Paskiewicz, D. M.; Sadeghirad, A.; Jakes, J.; Savage, D. E.; Stone, D. S.; Liu, F.; Lagally, M. A. Silicon nanomembranes as a means to evaluate stress evolution in deposited thin films. Extreme Mech. Lett. 2014, 1, 9-16.

18. Jamal, M.; Zarafshar, A. M.; Gracias, D. H. Differentially photo-crosslinked polymers enable self-assembling microfluidics. Nat. Comm. 2011, 2, 527.

19. Lee, J. N.; Park, C.; Whitesides G. M., Solvent compatibility of poly(dimethylsiloxane)-based microfluidic devices. Anal. Chem. 2003, 75(23), 6544-6554.

20. Braden, M.; Latham, D.; Patel, M. P. Observations on the swelling of cross-linked poly(dimethylsiloxane) networks by solvent. Eur. Polym. J. 2005, 41(12), 30693071.

21. Huan , L.; Guo , X.; Nuzzo , R. G.; Hsia , K. J. Capillary induced self-assembly of thin foils into 3D structures. J. Mech. Phys. Sol. 2010, 58, 2033-2042

22. Guo, X.; Li, H.; Ahna, B. Y.; Duoss, E. B.; Hsia, K. J.; Lewis, J. A.; Nuzzo, R. G. Two- and three-dimensional folding of thin film single-crystalline silicon for photovoltaic power applications. Proc. Nat. Acad. Sci. 2009, 106(48), 2014920154. 
23. Alben, S.; Balakrisnan, B.; Smela, E. Edge effects determine the direction of bilayer bending. Nano Lett. 2011, 11(6), 2280-2285.

24. Stoychev, G.; Zakharchenko, S.; Turcaud, S.; Dunlop, J. W. C.; Ionov, L. Shapeprogrammed folding of stimuli-responsive polymer bilayers. ACS Nano. 2012, 6 (5), 3925-3934.

25. Hyer, M. W. Calculations of the room-temperature shapes of unsymmetric laminates. J. Compos. Mater. 1981, 15, 296-310.

26. Shokrieh, M. M.; Kamali, S. M. Theoretical and experimental studies on residual stresses in laminated polymer composites. J. Compos. Mater. 2005, 39, 22132225.

27. Jeong, K.-U.; Jang, J.-H.; Kim, D.-Y.; Nah, C.; Lee, J. H.; Lee, M.-H.; Sun, H.-J.; Wang, C.-L.; Cheng, S. Z. D.; Thomas, E. L. Three-dimensional actuators transformed from the programmed two-dimensional structures via bending, twisting and folding mechanisms. J. Mater. Chem. 2011, 21, 6824-6830.

28. Stoychev, G.; Turcaud, S.; Dunlop, J. W. C.; Ionov, L. Hierarchical multi-step folding of polymer bilayers. Adv. Funct. Mater. 2013, 23(18), 2295-2300.

29. James, M. E. Polymer Data Handbook. Oxford Univeristy Press: New York, 1999.

30. Gundogan, N.; Okay, O.; Oppermann, W. Swelling, elasticity and spatial inhomogeneity of poly( $\mathrm{N}, \mathrm{N}$-dimethylacrylamide) hydrogels formed at various polymer concentrations. Macromol. Chem. Phys. 2004, 206(6), 814-823.

31. Parthasarathy, R.; Misra, A.; Park, J.; Ye, Q.; Spencer, P. Diffusion coefficients of water and leachables in methacrylate-based crosslinked polymers using absorption experiments. J. Mater. Sci. Mater. Med. 2012, 23(5), 1157-1172.

32. Armani, D.; Liu, C.; Aluru, N. In Micro Electro Mechanical Systems, Proceedings of the IEEE International Conference, Orlando, Fl, Jan 17-21, 1999; Reconfigurable fluid circuits by PDMS elastomer micromachining. 222-227.

33. Timoshenko, S. Analysis of Bi-Metal Thermostats. J.O.S.A \& R.S.I. 1925, 11(3), 233-255.

34. Madhukar, A.; Perlitz, D.; Grigola, M.; Gai, D.; Hsia, K. J. Bistable characteristics of axisymmetric dome. Int. J. Sol. Struct. 2014, 51(14), 2590-2597. 
35. Lv, C.; Yu, H.; Jiang, H. Archimedean spiral design for extremely stretchable interconnects. Extreme Mech. Lett. 2014, 1, 29-34. 


\section{Figure 1}

(a)
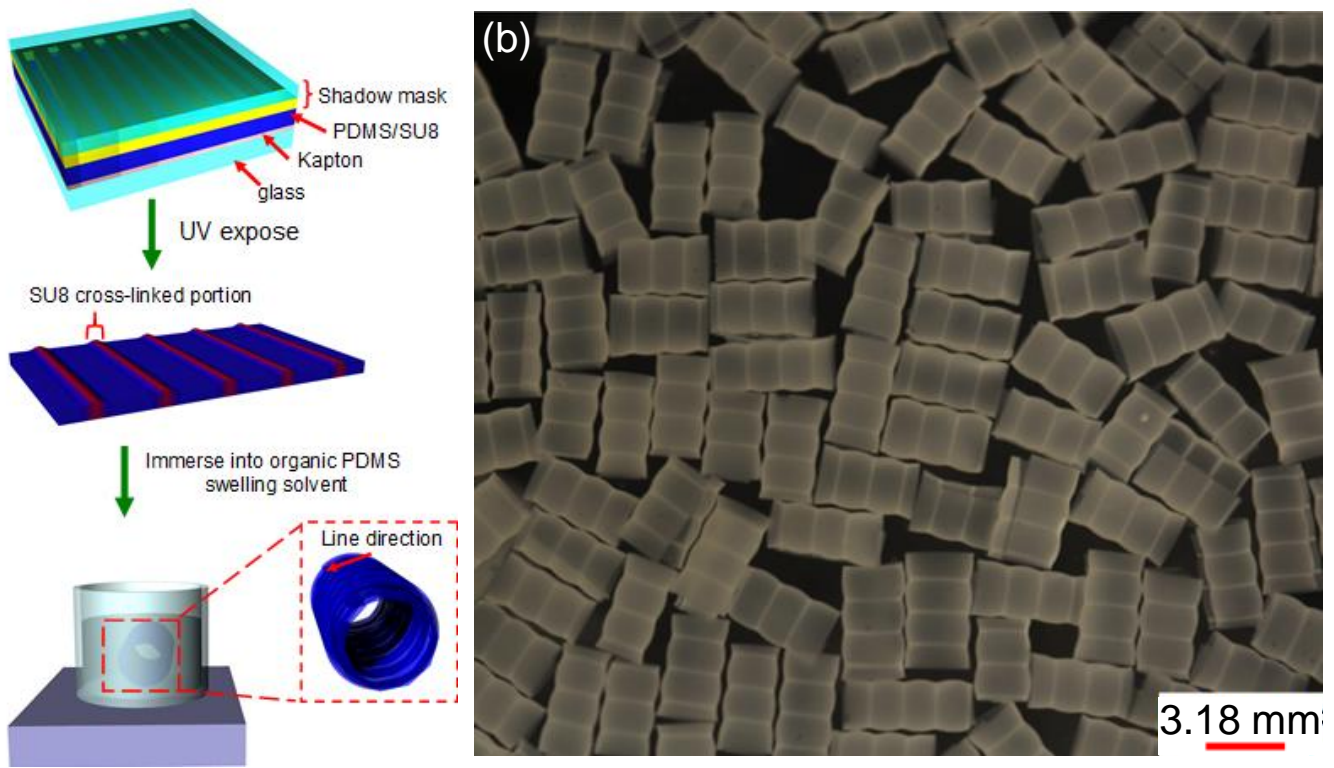


\section{Figure 2}

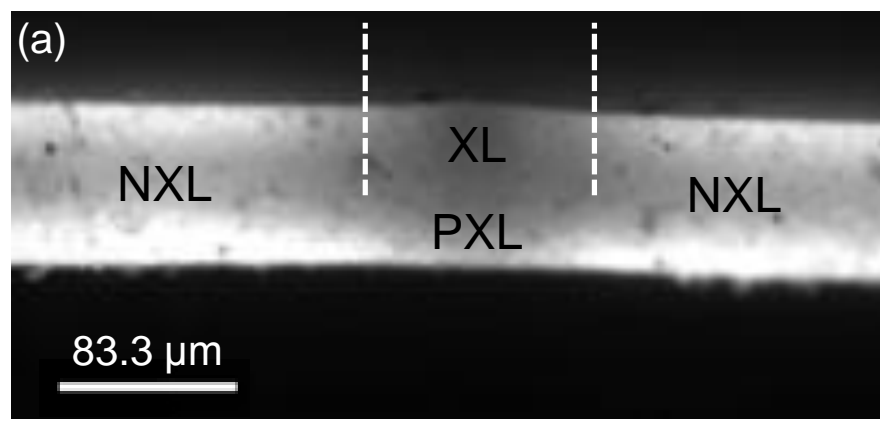

(b) Experimental profile
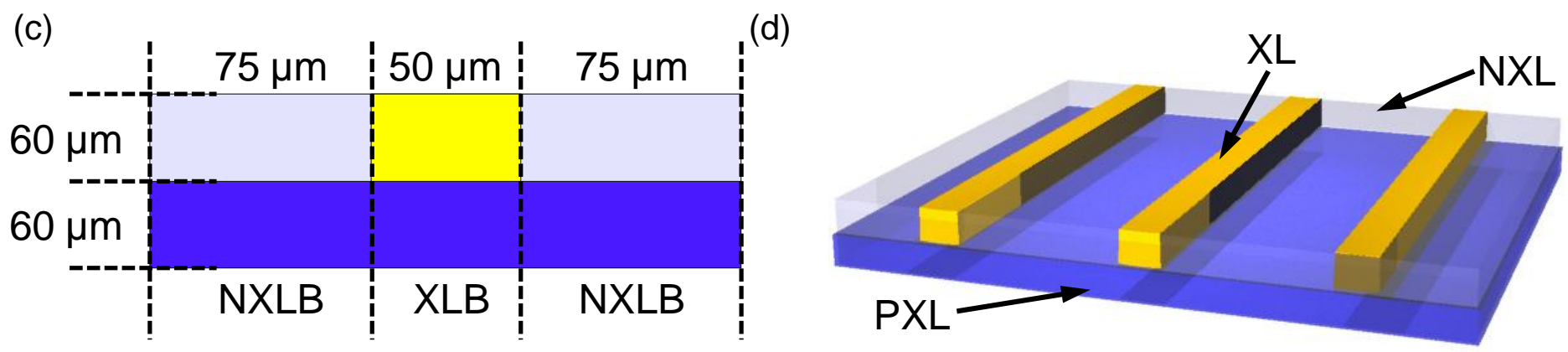


\section{Figure 3}

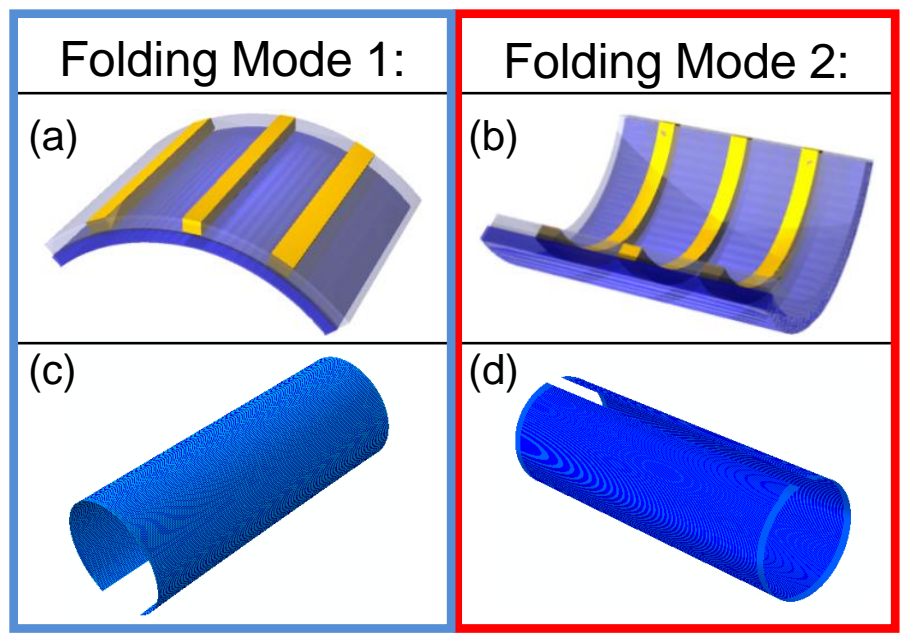




\section{Figure 4}

(a)
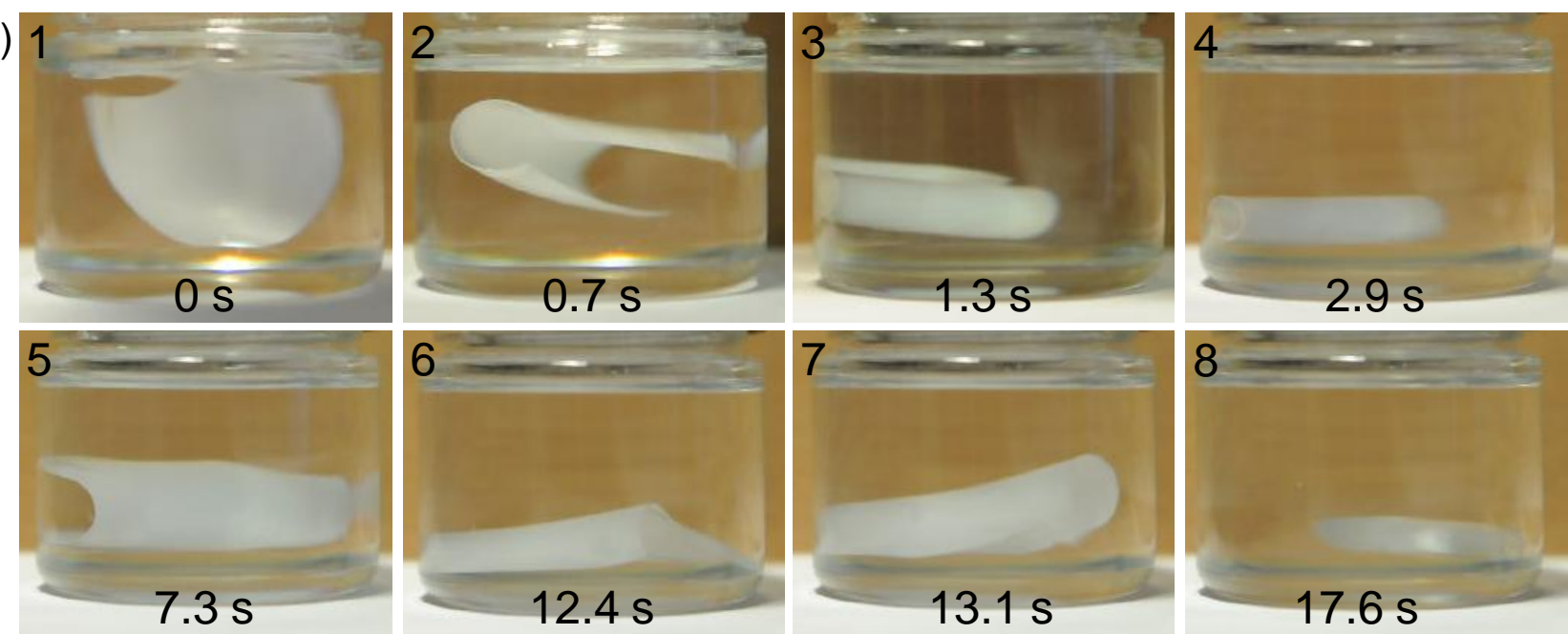

(b)

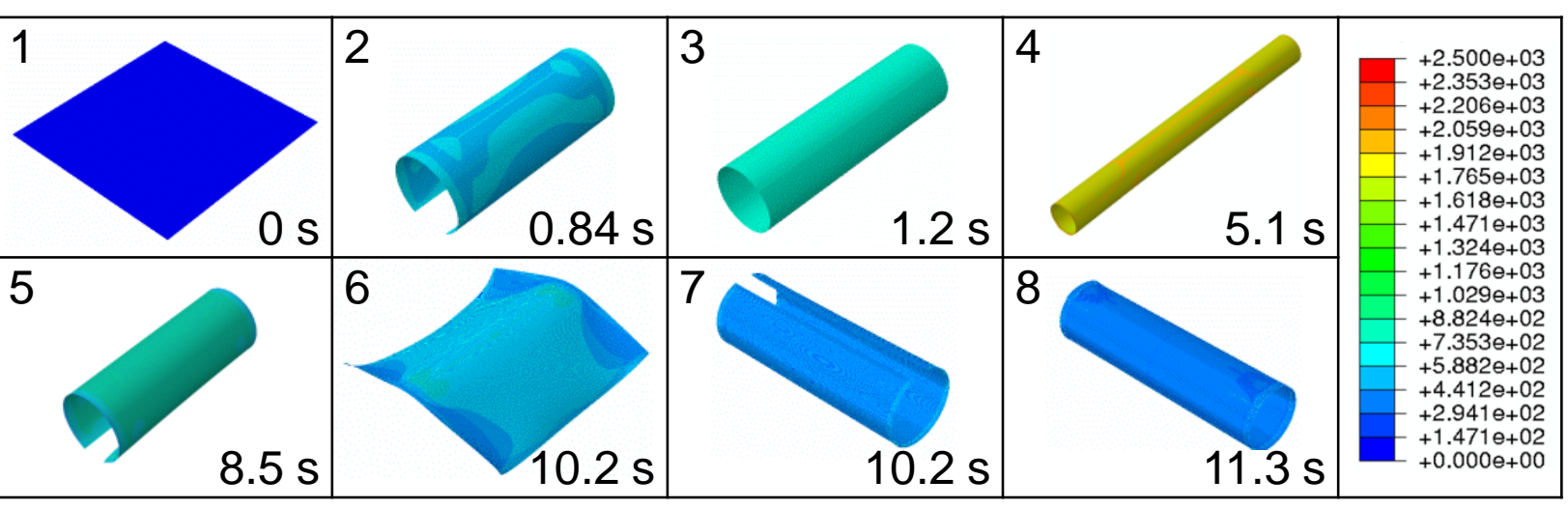




\section{Figure 5}

Folding Direction vs. Swelling Strain

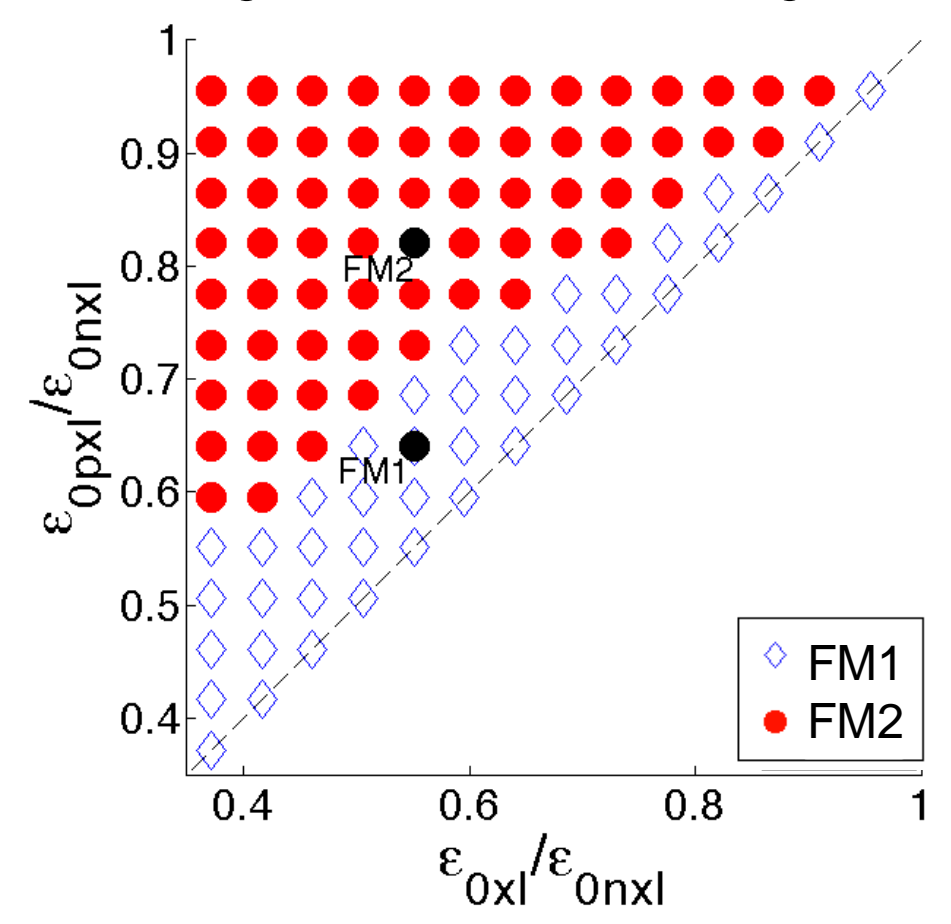




\section{Figure 6}

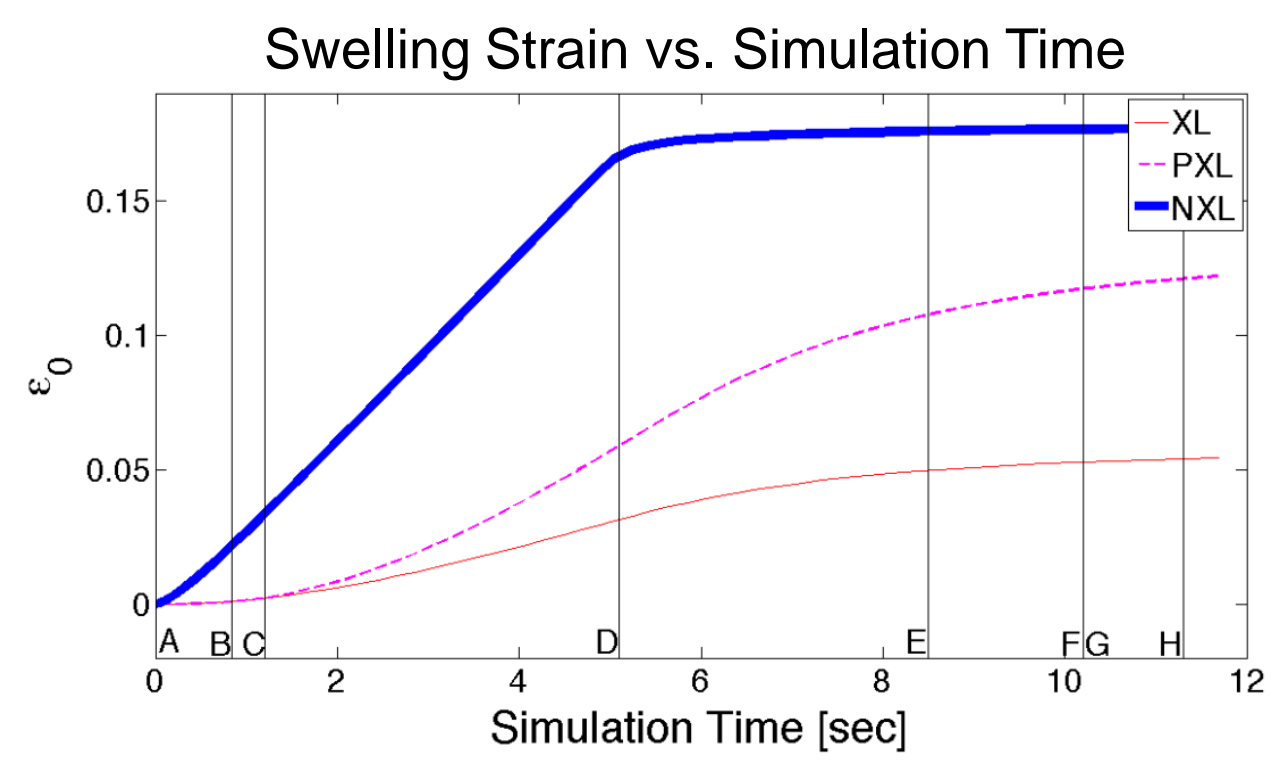




\section{Figure 7}

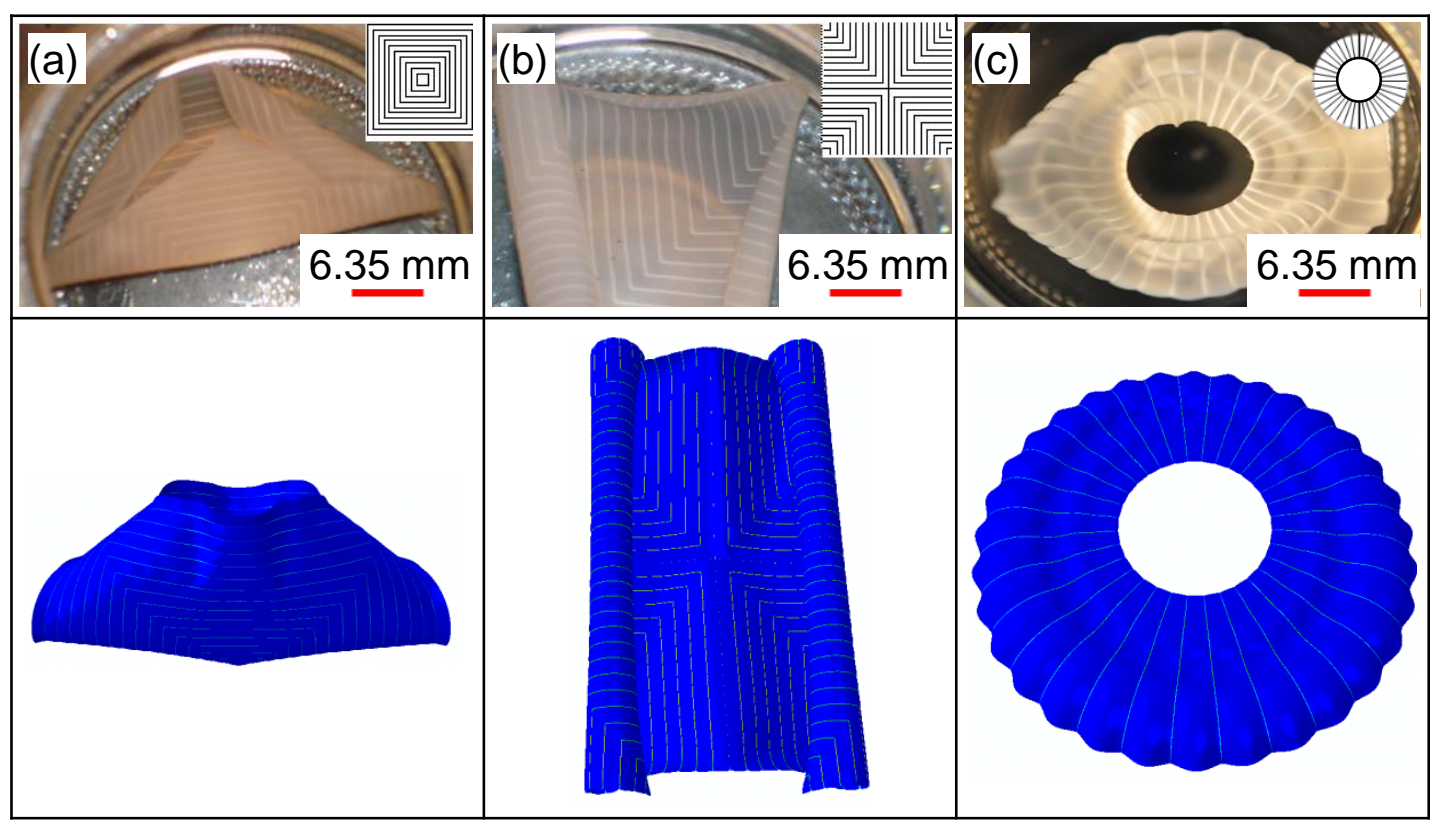

